\title{
Quantitative evaluation of small fatigue crack deflection behavior in metals based on T-stress
}

\author{
Jinta Arakawa ${ }^{1}$, Hiroyuki Akebono ${ }^{1 *}$, and Atsushi Sugeta ${ }^{1}$ \\ ${ }^{1}$ Department of Mechanical Science and Engineering, Hiroshima University, \\ 1-4-1 Kagamiyama, Higashi-Hiroshima, Hiroshima, Japan \\ *akebono@hiroshima-u.ac.jp
}

Introduction. The stage of fatigue crack propagation can be explained by using stage I, stage II, and stage III suggested by Forsyth et $\mathrm{al}^{(1)}$. Then, in our study, we focused on the fatigue crack propagation in the stage I, whose crack propagation depends on the single slip due to activation of slip plane on each material; for example, FCC material has 12 slip systems. Furthermore, there are very few references for fatigue crack deflection behavior on metal material. Therefore, in our study, we set that the aim is to suggest quantitative evaluation method by using stress state considering T-stress around the crack.

Experimental procedure. We used the $\alpha$-brass in the fatigue tests, and annealed under the condition of 873 $\mathrm{K}, 1$ hour, before cooling to the temperature in room. Furethermore, specimen is made by discharging mechanical machining for flat bar. Then, specimen surface is polished by emery papaer (\#800-\#2000), alminum pawder (3.0CR-0.1CR), and electrical polish $(1.2 \mathrm{~V}, 5 \mathrm{~s})$, in order to get the EBSD map in detail. The fatigue tests were carried out using plane bending fatigue tests machine, unedr the conditions of $R=-1$, $f=20 \mathrm{~Hz}, \sigma_{a}=50 \mathrm{MPa}$.

Quantitative evaluation method. The fatigue crack deflection was evaluated by using stress state around the fatigue crack, which acts on 4 slip planes of $\alpha$-brass. Furthermore, the stress state in arouud the crack tip can be expressed in Eq. (1). Then, Eq. (1) can be followed in Eq. (2), when we consider the mode I. The T-stress is the secound of Eq. (2), and acts on perpendicure direction of loading axis. The fatigue crack deflection behavior was evaluated using Eq. (2), and T-stress was calculated in stress difference method ${ }^{(2)}$.

$$
\begin{aligned}
\lim _{r \rightarrow 0} \sigma_{i j} & =\lim _{r \rightarrow 0}\left\{\left(\frac{k}{\sqrt{r}}\right) f_{i j}(\theta)+\sum_{m=0}^{\infty} A_{m} r^{m / 2} g_{i j}(m)(\theta)\right\} \\
& =\lim _{r \rightarrow 0}\left(\frac{k}{\sqrt{r}}\right) f_{i j}(\theta) \\
\sigma_{i j} & =\left(\frac{K}{\sqrt{2 \pi r}}\right) f_{i j}(\theta)+\left[\begin{array}{ccc}
T & 0 & 0 \\
0 & 0 & 0 \\
0 & 0 & v T
\end{array}\right]
\end{aligned}
$$

Experimental results. The fatigue crack propagation behavior indicated in Fig. 1, and it deflacted from B to $\mathrm{A}$ to $\mathrm{C}$ direction. The stress state unconsidering $\mathrm{T}$ stress in point 1 on $r_{1}$ direction was calculated by following Eq. (2), the results are indicated in Fig. 2. According to this figure, the stress state of defraction sngle 'A'is much lower than other stress states. Therfore, it is difficult to evaluate the fatigue crack deflection based on unconsidering T-stress. Then, in the next step, the stress state considering T-stress was calculated by following Eq. (2). The results are shown in Fig. 3. According to this figure, the stress state in slip angle ' $A$ ' is higher following to angle ' $B$ '. Therefore, it is clear that the slip angle ' $A$ ' is activative compared with angles 'C' and 'D'. So, it can be thought that the slip system 'A' acts after the alip system of 'B' is not activative.

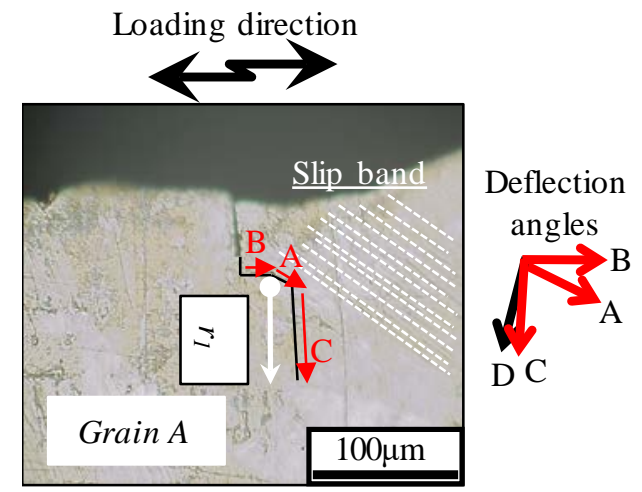

Fig. 1 Schematic illustration of the fatigue crack.

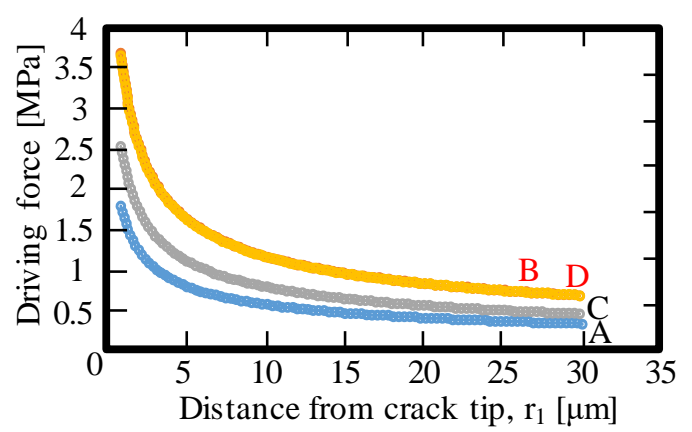

Fig. 2 Stress states around crack tip as a function of $r_{1}$ (Un-considering T-stress).

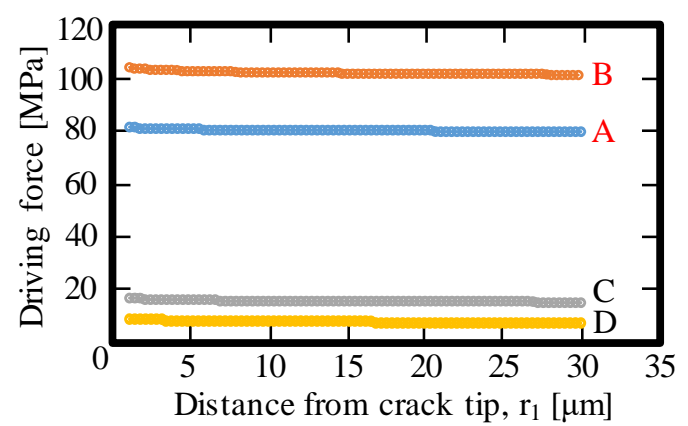

Fig. 3 Stress states around crack tip as a function of $r_{1}$ (Considering T-stress).

References.

(1) P, J, E, Forsyth., Acta Metallurgica, 11, (1963), 703-715

(2) M, Gupta., Eng Fract Mech, 134, (2015), 218-241 